\title{
Identification of vasospasm biomarkers for cerebral hemorrhage via bio-informatics analysis
}

\author{
Li-Ya Wu ${ }^{1}$, Yu-Bing Liang ${ }^{1}$, Sheng-Yu Li ${ }^{2}$, Guan-Feng Xie ${ }^{1}$, Yu-Jia Mei ${ }^{3}$, Chao Qin ${ }^{1}$ \\ ${ }^{1}$ Department of Neurology, the First Affiliated Hospital of Guangxi Medical University, Nanning, China; ${ }^{2}$ Department of Neurology, Wuming \\ Hospital, Guangxi Medical University, Nanning, China; ${ }^{3}$ Department of Neurology, the Second Affiliated Hospital of Guangxi Medical University, \\ Nanning, China \\ Contributions: (I) Conception and design: LY Wu, C Qin; Administrative support: YB Liang, SY Li; (III) Provision of study materials or patients: LY \\ Wu; (IV) Collection and assembly of data: GF Xie; (V) Data analysis and interpretation: YJ Mei; (VI) Manuscript writing: All authors; (VII) Final \\ approval of manuscript: All authors. \\ Correspondence to: Chao Qin. Department of Neurology, the First Affiliated Hospital of Guangxi Medical University, Nanning, China. \\ Email: qc20211618@126.com.
}

Background: The incidence of cerebral hemorrhage has rapidly increased over time, and vascular dysfunction has a significant influence on the pathogenesis and outcome of these patients. This is also the case for vasospasm in cerebral hemorrhage, but there is no method to assess this. We conducted this study to find molecular biomarkers of vasospasm in cerebral hemorrhage patients.

Methods: Raw data of GSE37924 was downloaded from the Gene Expression Omnibus (GEO) database, including 66 samples with cerebral vasospasm and 62 samples without cerebral vasospasm. Differentially expressed genes (DEGs) between samples with cerebral vasospasm and those without cerebral vasospasm were analyzed using the limma package in $\mathrm{R}$ software. To determine the functions of DEGs, we conducted functional enrichment analysis of DEGs through the clusterProfiler package in R. The protein-protein interaction (PPI) network of DEGs was constructed through STRING (https://string-db.org/) and generated via Cytoscape software. To understand the correlation between DEGs and immune-related genes, immune-related cerebral vasospasm genes were obtained via intersecting immune-related genes and cerebral vasospasm DEGs. We also compared the infiltration of 28 immune cells between cases with cerebral vasospasm and those without cerebral vasospasm. Finally, we constructed a model to perform the validation experiments.

Results: Of the DEGs, there were 24 upregulated and 21 downregulated genes in the vasospasm samples compared to the no-vasospasm samples. Functional enrichment analysis showed that these genes play key roles in several biological processes and signaling pathways such as the bone morphogenetic protein (BMP) signaling pathway, cellular response to BMP stimulus, natural killer cell chemotaxis, negative regulation of transmembrane receptor protein serine/threonine kinase signaling pathway, MHC protein complex binding, and receptor ligand activity, among others. CCL4, HLA-DQA1, IGF2, NTS, and so on were the significant immune-related genes. Furthermore, the immune cell infiltration results showed that there were differences between patients with vasospasm and those without vasospasm. Finally, we found that CCL4 had significantly higher expression in patients with vasospasm than those without vasospasm.

Conclusions: CCL4 is an important regulator of vascular dysfunction in cerebral hemorrhage.

Keywords: CCL4; cerebral hemorrhage; vasospasm

Submitted Nov 15, 2021. Accepted for publication Jan 18, 2022.

doi: 10.21037/apm-21-3717

View this article at: https://dx.doi.org/10.21037/apm-21-3717 


\section{Introduction}

Cerebral hemorrhage is a form of stroke which is characterized by high incidence, high risk of disability, and a high mortality rate. Cerebral hemorrhage accounted for $1 / 3$ of stroke cases worldwide in 2013 (1). There are several types of cerebral hemorrhage, including intracerebral hemorrhage ( $\mathrm{ICH})$, subarachnoid hemorrhage (SAH), and intraventricular hemorrhage (IVH). The main cause of cerebral hemorrhage is vascular disruption. Furthermore, cerebral hemorrhage can induce blood-brain barrier (BBB) dysfunction, and this process plays a significant role in brain damage after initial cerebral hemorrhage. BBB dysfunction will lead to circulating leukocyte extravasation and enhances brain injury $(2,3)$. All of these forms of cerebral hemorrhage cause increased BBB permeability, brain edema, and neuroinflammation. The vasculature is critical to cerebral hemorrhage but also plays a significant role after the initial hemorrhage, since it forms an integral part of the BBB.

There are several mechanisms involved in endothelial or vascular dysfunction. The main alterations occur in the junctions [particularly tight junctions (TJs)], which are significant regulators of endothelial cell death and transcytosis. However, only few studies have investigated the changes in vascular function after cerebral hemorrhage.

Several studies have examined brain endothelial junctions in ischemia but not in cerebral hemorrhage (4). In various cerebral hemorrhage types, many changes to the endothelium or vasculature can occur, with the main changes occurring in endothelial cell junctions. ZO-1, claudin-5, and occludin protein expression levels have been shown to decrease 1-3 days after ICH (5-10). All of these proteins are TJ proteins. Furthermore, brain claudin- 5 and occludin mRNA expression levels were shown to be downregulated 1-3 days after ICH (7). SAH can lead to reductions in cerebral blood flow, which can be influenced by intracranial pressure, hemorrhage mass effect, and macro/microvascular vasospasm (11). TJ proteins have also been detected in SAH (12-14). Cytoskeletal abnormal alterations have been found in smooth muscle cells after $\mathrm{SAH}$, and the underlying mechanism may be a change in the expression of S-100 (15). Except for cerebral hemorrhage, TJ proteins and mRNA expression levels have been reported to be altered in ischemia (4). Based on the fact that endothelial and vascular functions are critical for cerebral hemorrhage, studies have explored therapy targets after hemorrhage (16). However, most of these studies only focused on TJ protein expression and the endothelial cell junction, rather than vasospasm. We therefore performed this study to investigate the biomarkers of vasospasm after cerebral hemorrhage, aiming to further understand vascular regulation after cerebral hemorrhage and find novel therapies for the treatment of vascular dysfunction. Besides, we also analyzed the relationship between vasospasm and immune cell infiltration in cerebral hemorrhage in an attempt to find a new target for immunotherapy. We present the following article in accordance with the STREGA reporting checklist (available at https://apm. amegroups.com/article/view/10.21037/apm-21-3717/rc).

\section{Methods}

\section{Raw data}

The transcriptome data and clinical data of GSE37924 was downloaded from the Gene Expression Omnibus (GEO, https://www.ncbi.nlm.nih.gov/geo/) database, including 128 cases with cerebral hemorrhage. Of these, there were 66 cases with cerebral vasospasm and 62 cases without cerebral vasospasm. The study was conducted in accordance with the Declaration of Helsinki (as revised in 2013).

\section{Acquisition of differentially expressed genes (DEGs) related to cerebral vasospasm}

Analysis of DEGs between cases with cerebral vasospasm and those without cerebral vasospasm were performed in $\mathrm{R}$ software via the limma package, and $\log 2$ fold change $|\log \mathrm{FC}|>0.4$ and false discovery rate $(\mathrm{FDR})<0.05$ were the criteria for determining DEGs.

\section{Functional enrichment analysis of DEGs}

We conducted Gene Ontology (GO) and Kyoto Encyclopedia of Genes and Genomes (KEGG) functional enrichment analysis of DEGs through the clusterProfiler package in $\mathrm{R}$, and the results were presented via the ggplot2 package in $R$.

\section{Construction of the protein-protein interaction (PPI) network}

The PPI network of DEGs was constructed through the STRING database (https://string-db.org/) and generated via Cytoscape software. 


\section{Acquisition of immune genes associated with cerebral vasospasm}

An immune-related gene list was downloaded from the ImmPort database, and immune-related cerebral vasospasm genes were obtained by intersecting immune-related genes and cerebral vasospasm DEGs. We then analyzed the expression levels of immune-related DEGs in patients with and without cerebral vasospasm.

\section{Immune infiltration analysis}

We used single sample gene set enrichment analysis (ssGSEA) to calculate the immune infiltration landscapes of the GSE37924 cohort. The ssGSEA method is a machine learning algorithm used to characterize intratumoral immune landscapes and can identify the expression of 28 kinds of immune cells (17). We compared the infiltration of 28 immune cells between cases with cerebral vasospasm and those without cerebral vasospasm. Furthermore, we also analyzed the relationship between the abundance of the 28 immune cells and the expression of immune-related cerebral vasospasm genes.

\section{Validation experiments}

Establishment and evaluation of cerebrovascular spasm Rat brain hemorrhage models were established as previously described (18) using the non-anticoagulant autologous artery blood injection model. After weighing, rats were injected with $10 \%$ hydrated chloroform for anesthesia and fixed in the brain stereo positioning instrument (Shenzhen Ruiford Life Technology Co., Ltd., China), and the right caudate nucleus was localized according to the Rat Stereotaxic Atlas. The map was used to perform right tail nuclear positioning: a $1 \mathrm{~cm}$ longitudinal incision was made in the middle of the head to expose the fontanelle, the threedimensional positioning instrument was adjusted so that the front was $1 \mathrm{~mm}$ after $1 \mathrm{~mm}$, the right side was $3 \mathrm{~mm}$, and a small hole of about $1 \mathrm{~mm}$ in diameter was created direct to the brain (19). The right femoral artery was fully exposed, the syringe puncture was traced, and non-anticondensate artery blood $(100 \mu \mathrm{L})$ was extracted. The brain was slowly entered by drilling, slowly injected at $10 \mu \mathrm{L} / \mathrm{min}$, after the blood is completed, $10 \mathrm{~min}$, twice Neither, prevent blood return, medical bone wax to enclose drilling. After observing bleeding cessation, the scalp and the groin area incisions were monitored, and the rats were kept warm postoperatively until recovery. After resuscitation, the rats were scored according to the Longa method (20). 0 point: no nerve function; 1 point: the opposite front limb could not be completely stretched; 2 points: to the opposite side of the circle; 3 points: dump on the opposite side; 4 points: loss of consciousness. A score of 1 to 3 was considered model construction successful. At 72 hours after model establishment, the brain was removed from the head, and hematoma formation in the basal nucleus is found to be formed.

\section{Base artery disease test}

After model establishment, rats were administered with anesthesia, perfused with saline, depressed with $4 \%$ polymethylaldehyde ( $\mathrm{pH} 7.4$ ), and the complete brain base artery (BA) and middle cerebral artery (MCA) were obtained. After incubation at $4{ }^{\circ} \mathrm{C}$ in $4 \%$ paraformaldehyde overnight, tissues were dehydrated and embedded in paraffin. In order to avoid arterial branches, the BA was cross-cut at the same intermediate position $(2 \mathrm{~mm})$ each time. Every 4 slices cut in the coronal plane was stained with hematoxylin and eosin ( $\mathrm{HE}$ ) and observed under the microscope. The basilar arteries in the no-vasospasm group were found that the tube walls were thin, the lumen was large, and the endometrium was smooth. Angiogenesis of the base artery was visible, the tube wall was thickened, the lumen was small, endothelial cell deformation, and tortuous intimal folds were observed in vasospasm group.

\section{RNA extraction and quantitative real-time polymerase chain reaction (qRT-PCR)}

The total RNA of rat brain vascular tissues was extracted using TRIzol and RNAISO Plus Total RNA Extraction reagents (Japan Takara). The concentration and purity of RNA samples were measured by NanoDrop 2000 (Thermo Fisher Scientific, USA). Reverse transcription was performed with a random primer, and removal of genomic DNA was performed with the Primescript TMRT Reagent Kit and the GDNA ERASER kit (Japan Takara). Then, cDNA amplification was performed using TB Green Premix EX TAQ II (Japan, Takara) and the Applied Biosystems 7500 Fast Real Time PCR Systems Sequence Detection System (US Applied Biosystems). GAPDH and $\beta$-actin were used as internal controls, and each experiment was performed in triplicate. The relative quantification of mRNA expression was compared to internal parametrin, and the $2^{-\Delta \Delta \mathrm{Ct}}$ method was used. Finally, we performed validation of the selected genes to confirm the gene 

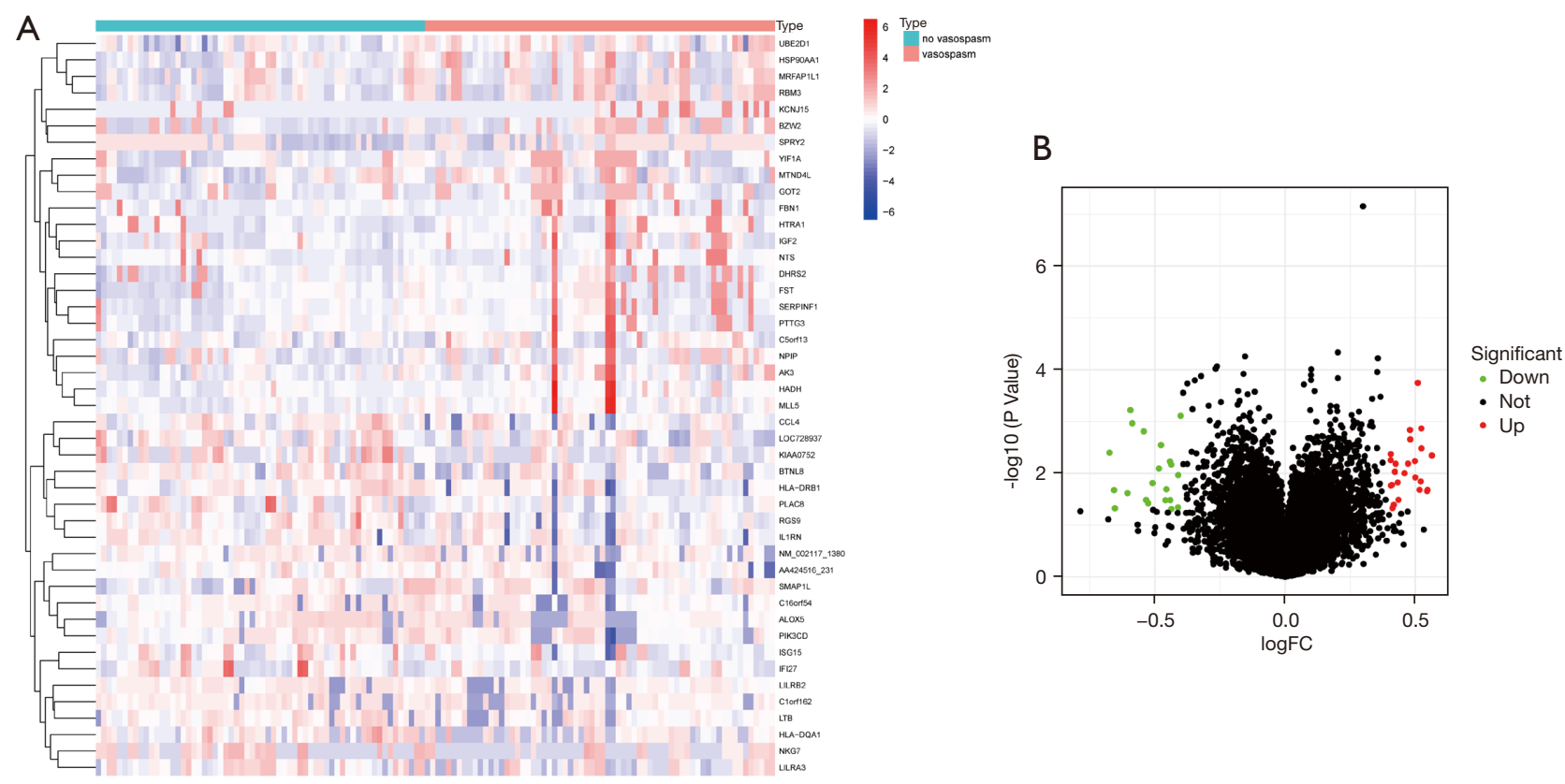

Figure 1 DEGs in cerebral hemorrhage patients. (A) Heatmap of DEGs in cerebral hemorrhage patients; (B) volcano plot of all DEGs, where red and green represent upregulated and downregulated genes, respectively. DEGs, differentially expressed genes.

expression levels.

\section{Ethical statement}

Experiments were performed under a project license (No. 202110002) granted by institutional ethics board of the First Affiliated Hospital of Guangxi Medical University, in compliance with the First Affiliated Hospital of Guangxi Medical University guidelines for the care and use of animals.

\section{Statistical analysis}

All statistical analyses were performed in R software. Difference analysis and correlation analysis were performed using the Wilcoxon test and Spearman correlation analysis, respectively. $\mathrm{P}<0.05$ was considered statistically significant.

\section{Results}

\section{Analysis of DEGs}

DEG analysis indicated that compared with cerebral hemorrhage patients without vasospasm, there were 24 upregulated and 21 downregulated genes in the vasospasm samples (Figure 1).

\section{Functional enrichment analysis results of DEGs}

The GO functional enrichment analysis results of DEGs indicated that negative regulation of transmembrane receptor protein serine/threonine kinase signaling pathway, $\mathrm{BMP}$ signaling pathway, response to BMP, cellular response to BMP stimulus, and natural killer cell chemotaxis were the top 5 biological processes (BP) in cerebral hemorrhage patients. COPII-coated ER to golgi transport vesicle, MHC class II protein complex, MHC protein complex, integral component of lumenal side of endoplasmic reticulum membrane, and lumenal side of endoplasmic reticulum membrane were the top 5 cell components (CC). Receptor ligand activity, signaling receptor activator activity, MHC protein complex binding, MHC class II receptor activity, and MHC class II protein complex binding were the top 5 molecular functions (MF) in cerebral hemorrhage patients (Figure 2A,2B).

The results of KEGG pathway analysis indicated that Epstein-Barr virus infection, antigen processing and presentation, B-cell receptor signaling pathway, rheumatoid 

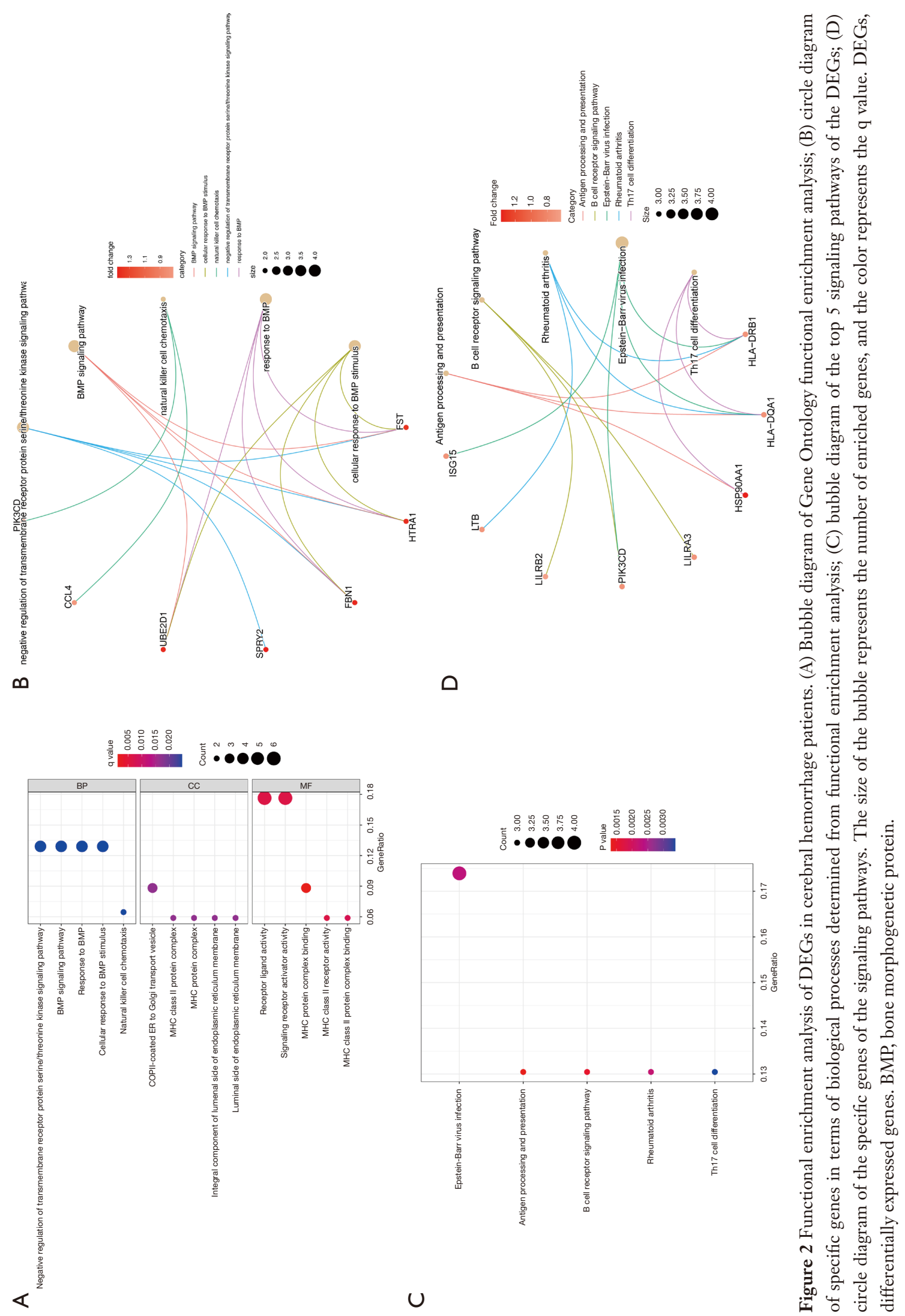


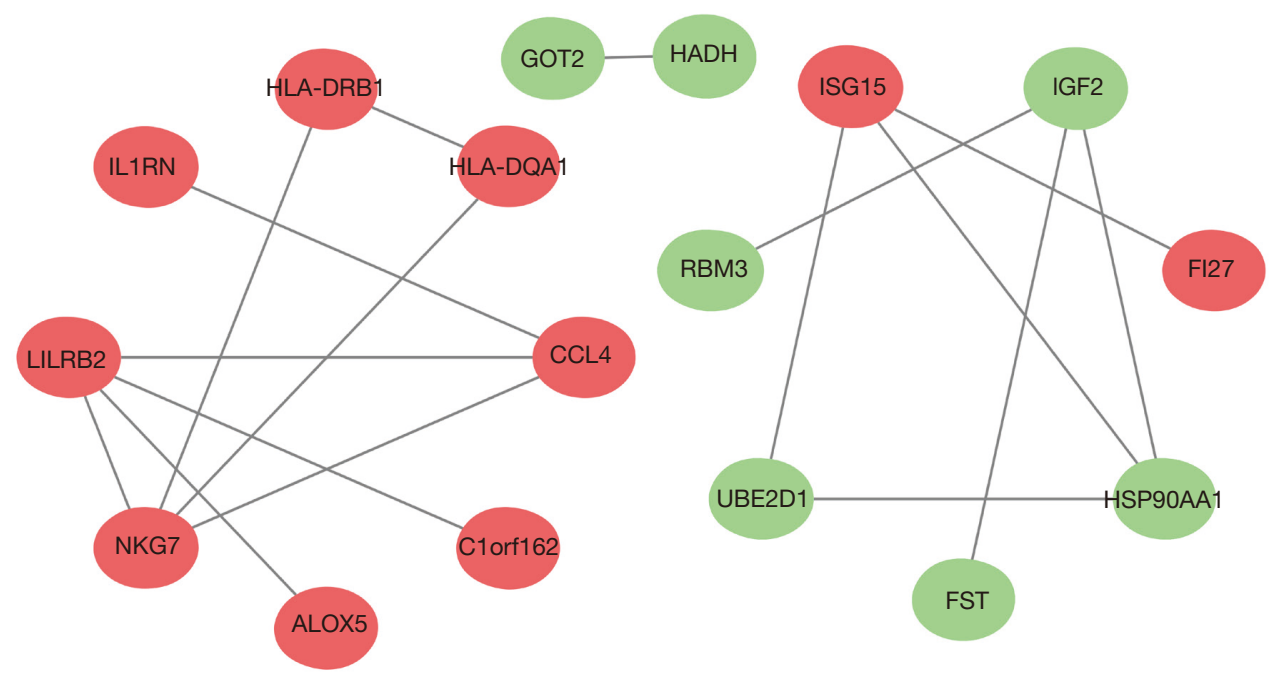

Figure 3 The PPI network of DEGs, where red and green represent upregulated and downregulated genes, respectively. PPI, proteinprotein interaction; DEGs, differentially expressed genes.

A

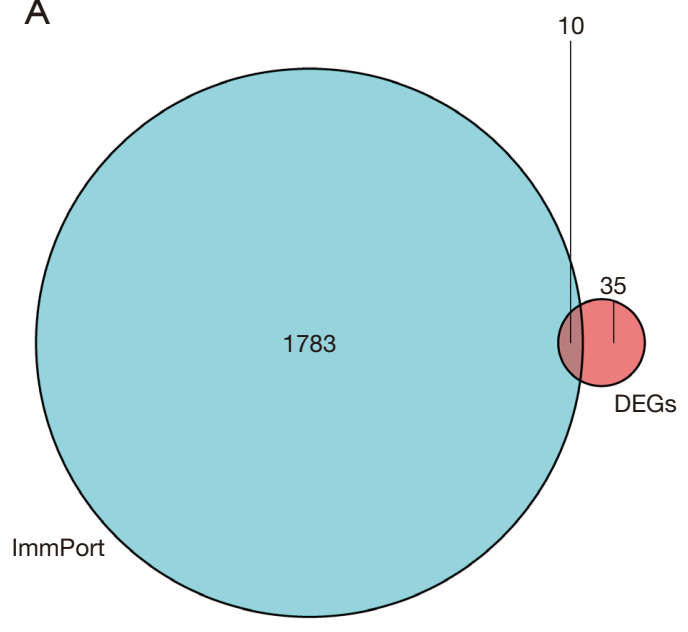

B

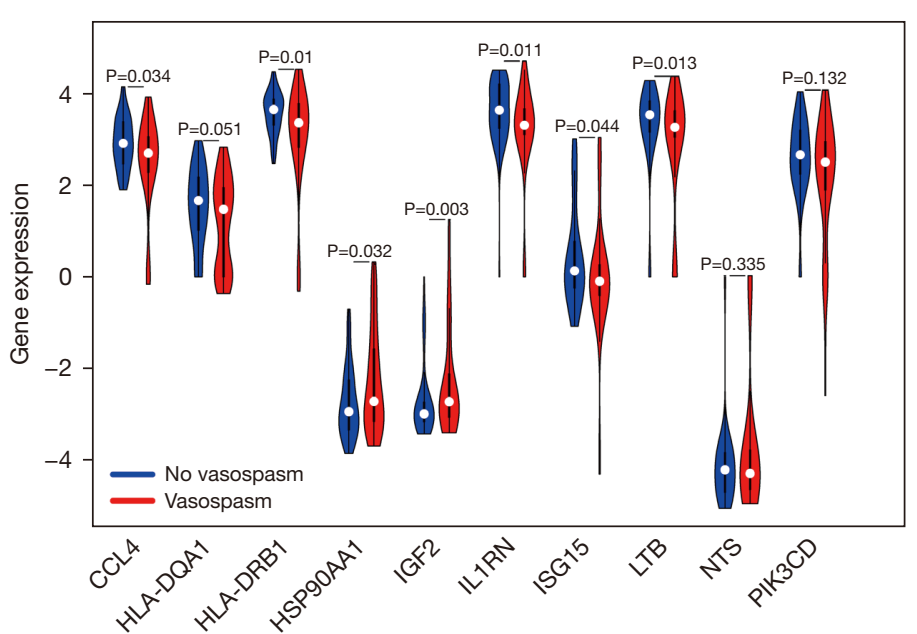

Figure 4 Immune-related genes associated with cerebral vasospasm. (A) Venn diagram of immune-related genes and DEGs; (B) expression levels of immune-related cerebral vasospasm genes. Blue and red represent no-vasospasm and vasospasm, respectively. DEGs, differentially expressed genes.

arthritis, and Th17 cell differentiation were the top 5 enriched KEGG pathways (Figure 2C,2D).

\section{The PPI network of DEGs}

We constructed a PPI network for 45 DEGs via the STRING database. The results showed that there were 17 DEGs which interacted with each other, comprising 9 upregulated genes and 8 downregulated genes (Figure 3).

\section{Immune-related genes associated with cerebral vasospasm}

Venn analysis results revealed that there were 10 overlapping genes between immune-related genes and DEGs in cerebral vasospasm (Figure $4 A$ ). Differential expression analysis results showed that $\mathrm{C}-\mathrm{C}$ motif chemokine ligand 
4 (CCL4), major histocompatibility complex, class II, DQ alpha 1 (HLA-DQA1), major histocompatibility complex, class II, DR beta 1 (HLA-DRB1), interleukin 1 receptor antagonist (IL1RN), ISG15 ubiquitin like modifier (ISG15), and lymphotoxin beta $(L T B)$ were expressed at low levels in patients with cerebral vasospasm, while heat shock protein 90 alpha family class A member 1 (HSP90AA1) and insulin like growth factor 2 (IGF2) were expressed at high levels in cerebral vasospasm patients (Figure $4 B$ ).

\section{Levels of immune cell infiltration in cerebral hemorrhage patients}

Each of the 28 immune cells in the ssGSEA analysis are shown in a histogram (Figure $5 \mathrm{~A}$ ). Correlation analysis between immune cells showed that type $17 \mathrm{~T}$ helper cells and effector memory CD4 $\mathrm{T}$ cells were negatively correlated with the abundance of most other immune cells, while most of the other 26 types of immune cells were positively correlated (Figure 5B). Myeloid derived suppressor cells, neutrophils, effector memory CD8 T cells, immature $B$ cells, activated dendritic cells, and mast cells showed high levels of infiltration in cerebral hemorrhage patients (Figure 5C). Correlation analysis results demonstrated that the expression levels of CCL4, IL1RN, PIK3CD, HLA$D Q A 1, L T B, H L A-D R B 1$, and $I S G 15$ were positively correlated with the infiltration levels of most immune cells, while the expression levels of HSP90AA1, IGF2, and NTS were negatively correlated with the infiltration levels of most immune cells (Figure 5D).

\section{Differential gene expression levels}

The validation experiments showed that CCL4, Rasgpr1, and Spp1 were significantly expressed in vasospasm samples, while $V E G F C$, IL17B, HCST, and Crb1 were downregulated in vasospasm samples (Figure 6).

\section{Discussion}

Cerebral hemorrhage is a form of stroke characterized by high mortality. Risk factors of cerebral hemorrhage include hypertension and atherosclerosis, among others. The dysfunction of blood vessels has a critical influence on the treatment choice, and can even distinguish the outcomes of these patients. At present, there is a lack of sensitive and specific markers and imaging diagnostic tools to identify the patients with or without vasospasm. Several factors have a strong influence on vascular function during the initial stages or after cerebral hemorrhage, especially gene alterations $(4,15)$. Thus, we analyzed DEGs between patients with and without vasospasm, and performed enrichment analysis of the selected genes to determine their roles in cerebral vasospasm.

Of the DEGs found in our study, there were 24 upregulated and 21 downregulated genes in the vasospasm samples compared to those without vasospasm. Furthermore, we selected DEGs for validation experiments via the hub PPI network. Gene expression level results showed that only CCL4 was significantly expressed in vasospasm patients. CCL4, a CC chemokine, also known as macrophage inflammatory protein (MIP)$1 \beta$, has a variable influence on multiple immune and nonimmune cells via its interaction with its specific receptor, CCR5. Several studies have shown that the CCL4 signaling pathway is important for tumorigenesis and progression (21-24). Zhu et al. revealed that CCL4 may play a critical role in ischemic stroke, and may be significantly correlated with sex (25). CCL4 has also been shown to regulate human vascular smooth muscle cells (26). Furthermore, Chang et al. indicated that CCL4 can act as an inhibitor for atherosclerosis via regulating macrophage cells (27). However, there are no studies on the relationship between CCL4 and cerebral hemorrhage.

To determine the fundamental biological functions of these DEGs, we performed functional enrichment analysis. The results showed that these genes play key roles in several biological processes and signaling pathways such as the bone morphogenetic protein (BMP) signaling pathway, cellular response to BMP stimulus, natural killer cell chemotaxis, negative regulation of transmembrane receptor protein serine/threonine kinase signaling pathway, MHC protein complex binding, and receptor ligand activity, among others. The BMP signaling pathway has not been investigated in cerebral hemorrhage, but there are several studies that have demonstrated its critical function in various vascular diseases (28-30). The transmembrane receptor protein serine/threonine kinase signaling pathway also acts as the significant regulator for vascular (31). MHC class II antigen but not MHC protein complex binding has been detected in traumatic brain injury (32). According to natural killer cell chemotaxis, it is the immune cells that have been clarified in the cerebral hemorrhage. Kim et al. revealed that natural killer cell chemotaxis plays a significant role in delayed cerebral ischemia following SAH (33). From these findings, the DEGs found in this study play significant 

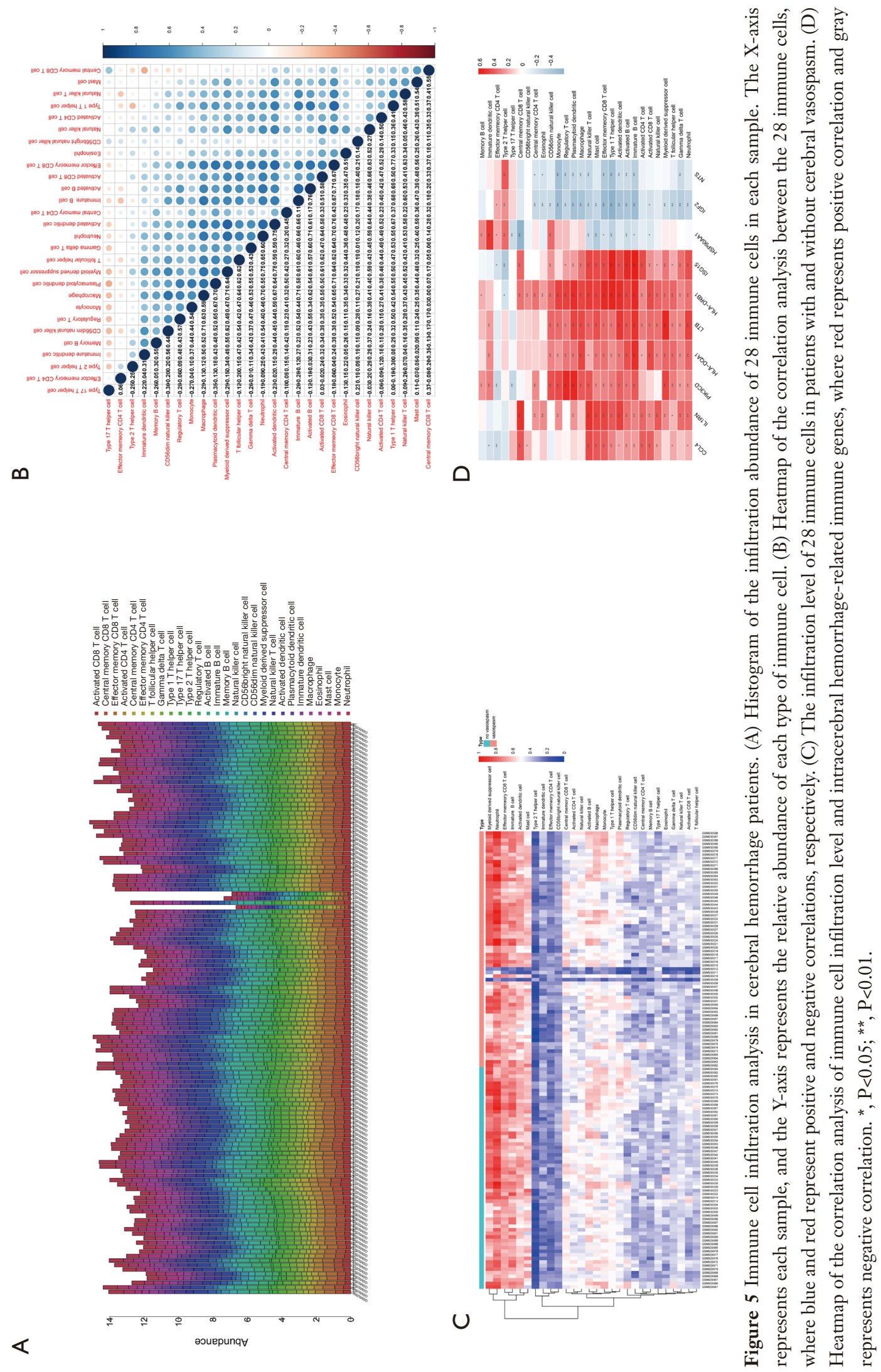

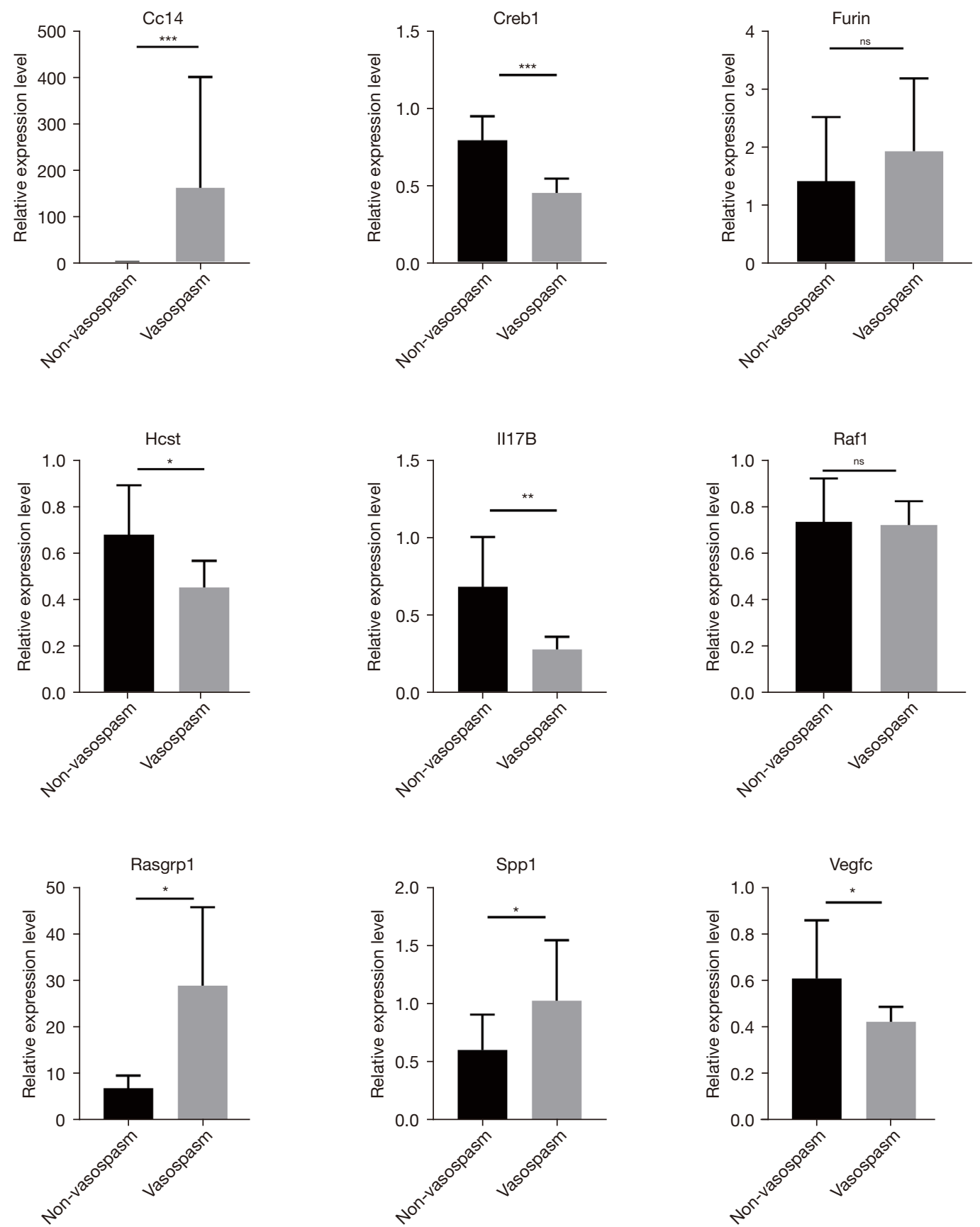

Figure 6 The PCR results of several genes between vasospasm and without vasospasm. The ns represents $\mathrm{P}>0.05$. ${ }^{*}, \mathrm{P}<0.05 ;{ }^{* *}, \mathrm{P}<0.01 ;{ }^{* * *}$, $\mathrm{P}<0.001$. PCR, polymerase chain reaction.

roles in the regulation of vascular diseases, but there is no research on the involvement of these DEGs in cerebral stroke.

Several studies have shown that inflammation plays a significant role in angiogenesis (34-36). The relationship between inflammation and the vasculature has been well investigated in cancer, but few studies have been performed in others diseases. Capitão et al. showed that inflammation has a significant influence on diabetic retinopathy (34). Inflammation is also strongly correlated with immunity. 
In this study, we conducted an analysis of the intersection between DEGs and immune-related genes, as immunerelated genes often work via the regulation of immune cell infiltration. We selected the intersecting immune-related genes and explored the relationships between immunerelated genes and the infiltration of various immune cells. The results showed that CCL4, HLA-DQA1, HLA-DRB1, HSP90AA1, IGF2, IL1R, NISG15, LTB, NTS, and PIK3CD were intersecting genes. LTB4 has been investigated for its role in cerebral hemorrhage (37-40). Hijioka et al. indicated that $L T B$ can promote neutrophil infiltration, leading to neuroinflammation (41). Zhu et al. showed that IGF2 could regulate vascular smooth muscle cells (42). IGF2 is also a novel target for psychiatric and neurological disorders (43). Durocher et al. showed that PIK3CD was the immune response hub gene for ICH (44). Our results showed that there were differences in immune cells between patients with and without vasospasm. Neutrophils and natural killer cells had higher infiltration in vasospasm patients. Our findings show that these immune-related genes and immune cells may act as significant factors in cerebral hemorrhage and vascular dysfunction such as vasospasm. Xue et al.' review indicated that in animal models and early clinical trials in intracerebral haemorrhage, several drugs have reduced detrimental neuroinflammation without substantial compromise of the beneficial reparative aspects of an inflammatory response (45). Other genes have been not yet been determined in cerebral hemorrhage. The limitation of this study is the lack of in vitro and in vivo experiments. Further, we will conduct vitro and in vivo experiments on the hub genes in present study to verify the relationship between gene expression regulation and cerebral vasospasm, so as to lay a foundation for accurate target therapy of cerebral hemorrhage.

\section{Conclusions}

The immune-related gene CCL 4 may act as a critical regulator of cerebral hemorrhage patients with vasospasm, but the fundamental biological function of this gene remains unclear. Our present study may pave the way for further research in vasospasm after cerebral hemorrhage.

\section{Acknowledgments}

Funding: This work was supported by National Natural Science Foundation of China (Nos. 81860222, 82060226) and Guangxi Natural Science Foundation (No.
2019GXNSFDA185008).

\section{Footnote}

Reporting Checklist: The authors have completed the STREGA reporting checklist. Available at https://apm. amegroups.com/article/view/10.21037/apm-21-3717/rc

Data Sharing Statement: Available at https://apm.amegroups. com/article/view/10.21037/apm-21-3717/dss

Conflicts of Interest: All authors have completed the ICMJE uniform disclosure form (available at https://apm. amegroups.com/article/view/10.21037/apm-21-3717/coif). The authors have no conflicts of interest to declare.

Ethical Statement: The authors are accountable for all aspects of the work in ensuring that questions related to the accuracy or integrity of any part of the work are appropriately investigated and resolved. The study was conducted in accordance with the Declaration of Helsinki (as revised in 2013). Experiments were performed under a project license (No. 202110002) granted by institutional ethics board of the First Affiliated Hospital of Guangxi Medical University, in compliance with the First Affiliated Hospital of Guangxi Medical University guidelines for the care and use of animals.

Open Access Statement: This is an Open Access article distributed in accordance with the Creative Commons Attribution-NonCommercial-NoDerivs 4.0 International License (CC BY-NC-ND 4.0), which permits the noncommercial replication and distribution of the article with the strict proviso that no changes or edits are made and the original work is properly cited (including links to both the formal publication through the relevant DOI and the license). See: https://creativecommons.org/licenses/by-nc-nd/4.0/.

\section{References}

1. Benjamin EJ, Blaha MJ, Chiuve SE, et al. Heart Disease and Stroke Statistics-2017 Update: A Report From the American Heart Association. Circulation 2017;135:e146-603.

2. Keep RF, Hua Y, Xi G. Intracerebral haemorrhage: mechanisms of injury and therapeutic targets. Lancet Neurol 2012;11:720-31.

3. Keep RF, Zhou N, Xiang J, et al. Vascular disruption 
and blood-brain barrier dysfunction in intracerebral hemorrhage. Fluids Barriers CNS 2014;11:18.

4. Jiang X, Andjelkovic AV, Zhu L, et al. Blood-brain barrier dysfunction and recovery after ischemic stroke. Prog Neurobiol 2018;163-164:144-71.

5. Krafft PR, Caner B, Klebe D, et al. PHA-543613 preserves blood-brain barrier integrity after intracerebral hemorrhage in mice. Stroke 2013;44:1743-7.

6. Li Z, Chen X, Zhang X, et al. Small Interfering RNA Targeting Dickkopf-1 Contributes to Neuroprotection After Intracerebral Hemorrhage in Rats. J Mol Neurosci 2017;61:279-88.

7. Sun H, Tang Y, Guan X, et al. Effects of selective hypothermia on blood-brain barrier integrity and tight junction protein expression levels after intracerebral hemorrhage in rats. Biol Chem 2013;394:1317-24.

8. Wang T, Chen X, Wang Z, et al. Poloxamer-188 can attenuate blood-brain barrier damage to exert neuroprotective effect in mice intracerebral hemorrhage model. J Mol Neurosci 2015;5 5:240-50.

9. Wanyong Y, Zefeng T, Xiufeng X, et al. Tempol alleviates intracerebral hemorrhage-induced brain injury possibly by attenuating nitrative stress. Neuroreport 2015;26:842-9.

10. Xie RX, Li DW, Liu XC, et al. Carnosine Attenuates Brain Oxidative Stress and Apoptosis After Intracerebral Hemorrhage in Rats. Neurochem Res 2017;42:541-51.

11. Tso MK, Macdonald RL. Subarachnoid hemorrhage: a review of experimental studies on the microcirculation and the neurovascular unit. Transl Stroke Res 2014;5:174-89.

12. Chen D, Wei XT, Guan JH, et al. Inhibition of c-Jun $\mathrm{N}$-terminal kinase prevents blood-brain barrier disruption and normalizes the expression of tight junction proteins clautin-5 and ZO-1 in a rat model of subarachnoid hemorrhage. Acta Neurochir (Wien) 2012;154:1469-76; discussion 1476.

13. Chen J, Chen G, Li J, et al. Melatonin attenuates inflammatory response-induced brain edema in early brain injury following a subarachnoid hemorrhage: a possible role for the regulation of pro-inflammatory cytokines. J Pineal Res 2014;57:340-7.

14. Fan R, Enkhjargal B, Camara R, et al. Critical role of EphA4 in early brain injury after subarachnoid hemorrhage in rat. Exp Neurol 2017;296:41-8.

15. Lefranc F, Golzarian J, Chevalier C, et al. Expression of members of the calcium-binding S-100 protein family in a rat model of cerebral basilar artery vasospasm. J Neurosurg 2002;97:408-15

16. Keep RF, Andjelkovic AV, Xiang J, et al. Brain endothelial cell junctions after cerebral hemorrhage: Changes, mechanisms and therapeutic targets. J Cereb Blood Flow Metab 2018;38:1255-75.

17. Charoentong P, Finotello F, Angelova M, et al. Pancancer Immunogenomic Analyses Reveal GenotypeImmunophenotype Relationships and Predictors of Response to Checkpoint Blockade. Cell Rep 2017;18:248-62.

18. MacLellan CL, Gyawali S, Colbourne F. Skilled reaching impairments follow intrastriatal hemorrhagic stroke in rats. Behav Brain Res 2006;175:82-9.

19. Paxinos G, Watson CR, Emson PC. AChE-stained horizontal sections of the rat brain in stereotaxic coordinates. J Neurosci Methods 1980;3:129-49.

20. Longa EZ, Weinstein PR, Carlson S, et al. Reversible middle cerebral artery occlusion without craniectomy in rats. Stroke 1989;20:84-91.

21. Hu GN, Tzeng HE, Chen PC, et al. Correlation between CCL4 gene polymorphisms and clinical aspects of breast cancer. Int J Med Sci 2018;15:1179-86.

22. Korbecki J, Grochans S, Gutowska I, et al. CC Chemokines in a Tumor: A Review of Pro-Cancer and Anti-Cancer Properties of Receptors CCR5, CCR6, CCR7, CCR8, CCR9, and CCR10 Ligands. Int J Mol Sci 2020;21:7619.

23. Korbecki J, Kojder K, Simińska D, et al. CC Chemokines in a Tumor: A Review of Pro-Cancer and Anti-Cancer Properties of the Ligands of Receptors CCR1, CCR2, CCR3, and CCR4. Int J Mol Sci 2020;21:8412.

24. Uehara T, Pogribny IP, Rusyn I. The DEN and CCl4 -Induced Mouse Model of Fibrosis and InflammationAssociated Hepatocellular Carcinoma. Curr Protoc Pharmacol 2014;66:14.30.1-10.

25. Zhu W, Nan Y, Wang S, et al. Bioinformatics Analysis of Gene Expression Profiles of Sex Differences in Ischemic Stroke. Biomed Res Int 2019;2019:2478453.

26. Wang J, Wu Q, Yu J, et al. miR-125a-5p inhibits the expression of NLRP3 by targeting CCL4 in human vascular smooth muscle cells treated with ox-LDL. Exp Ther Med 2019;18:1645-52.

27. Chang TT, Yang HY, Chen C, et al. CCL4 Inhibition in Atherosclerosis: Effects on Plaque Stability, Endothelial Cell Adhesiveness, and Macrophages Activation. Int J Mol Sci 2020;21:6567.

28. García de Vinuesa A, Abdelilah-Seyfried S, Knaus P, et al. BMP signaling in vascular biology and dysfunction. Cytokine Growth Factor Rev 2016;27:65-79.

29. Petersen MA, Ryu JK, Chang KJ, et al. Fibrinogen 
Activates BMP Signaling in Oligodendrocyte Progenitor Cells and Inhibits Remyelination after Vascular Damage. Neuron 2017;96:1003-1012.e7.

30. Soma Y, Sugishita M, Maruyama S, et al. Interhemispheric visual information transfer in callosal agenesis. No To Shinkei 1989;41:149-55.

31. Burch ML, Getachew R, Osman N, et al. Thrombinmediated proteoglycan synthesis utilizes both proteintyrosine kinase and serine/threonine kinase receptor transactivation in vascular smooth muscle cells. J Biol Chem 2013;288:7410-9.

32. Orihara $Y$, Ikematsu $K$, Tsuda $R$, et al. Induction of nitric oxide synthase by traumatic brain injury. Forensic Sci Int 2001;123:142-9.

33. Kim BJ, Youn DH, Chang IB, et al. Identification of Differentially-Methylated Genes and Pathways in Patients with Delayed Cerebral Ischemia Following Subarachnoid Hemorrhage. J Korean Neurosurg Soc 2022;65:4-12.

34. Capitão M, Soares R. Angiogenesis and Inflammation Crosstalk in Diabetic Retinopathy. J Cell Biochem 2016;117:2443-53.

35. Noonan DM, De Lerma Barbaro A, Vannini N, et al. Inflammation, inflammatory cells and angiogenesis: decisions and indecisions. Cancer Metastasis Rev 2008;27:31-40.

36. Whiteford JR, De Rossi G, Woodfin A. Mutually Supportive Mechanisms of Inflammation and Vascular Remodeling. Int Rev Cell Mol Biol 2016;326:201-78.

37. Broyet C, Guérin C, Pozzetto B, et al. Meningoencephalitis in Epstein-Barr virus reactivation in a renal transplant patient. Presse Med 1989;18:1168.

38. Hijioka M, Anan J, Ishibashi H, et al. Inhibition

Cite this article as: Wu LY, Liang YB, Li SY, Xie GF, Mei YJ, Qin C. Identification of vasospasm biomarkers for cerebral hemorrhage via bio-informatics analysis. Ann Palliat Med 2022;11(1):173-184. doi: 10.21037/apm-21-3717 of Leukotriene B4 Action Mitigates Intracerebral Hemorrhage-Associated Pathological Events in Mice. J Pharmacol Exp Ther 2017;360:399-408.

39. Yaqoob M, Ahmad R, Simkin E. Hypocalcemia after parathyroidectomy. Br Med J (Clin Res Ed) 1988;296:1198.

40. Zhang Z, Zhao G, Liu L, et al. Bexarotene Exerts Protective Effects Through Modulation of the Cerebral Vascular Smooth Muscle Cell Phenotypic Transformation by Regulating PPAR $\gamma /$ FLAP/LTB4 After Subarachnoid Hemorrhage in Rats. Cell Transplant 2019;28:1161-72.

41. Hijioka M, Futokoro R, Ohto-Nakanishi T, et al. Microglia-released leukotriene B4 promotes neutrophil infiltration and microglial activation following intracerebral hemorrhage. Int Immunopharmacol 2020;85:106678.

42. Zhu D, Mackenzie NC, Millan JL, et al. Upregulation of IGF2 expression during vascular calcification. J Mol Endocrinol 2014;52:77-85.

43. Pardo M, Cheng Y, Sitbon YH, et al. Insulin growth factor 2 (IGF2) as an emergent target in psychiatric and neurological disorders. Review. Neurosci Res 2019;149:1-13.

44. Durocher M, Ander BP, Jickling G, et al. Inflammatory, regulatory, and autophagy co-expression modules and hub genes underlie the peripheral immune response to human intracerebral hemorrhage. J Neuroinflammation 2019;16:56.

45. Xue M, Yong VW. Neuroinflammation in intracerebral haemorrhage: immunotherapies with potential for translation. Lancet Neurol 2020;19:1023-32.

(English Language Editor: C. Betlazar-Maseh) 